International Journal of Modern Physics E Vol. 24, No. 3 (2015) 1550020 (16 pages)

\title{
Relativistic and nonrelativistic solutions of the generalized Poschl-Teller and hyperbolical potentials with some thermodynamic properties
}

$$
\text { C. A. Onate }{ }^{1} * \text {, and J. O. Ojonubah }{ }^{2}
$$

${ }^{1}$ Physics Section, Kogi State Teaching Service Commission, ECSS Uchuchu, Ibaji, Lokoja, Nigeria

${ }^{2}$ Mathematics Department, Federal College of Education, Okene, Nigeria "oaclems14@physicist.net

Received 9 October 2014

Revised 27 December 2014

Accepted 6 February 2015

Published 19 March 2015

\begin{abstract}
By using the new approximation type, the Dirac equation is solved with the combination of Generalized P" oschl-Teller and Hyperbolical potentials within the framework of supersymmetric approach. The energy levels are obtained for both pseudospin and spin symmetries and the nonrelativistic limit is obtained with the corresponding wave functions in terms of hypergeometric functions. Some thermodynamic properties are equally obtained with the energy equation of the nonrelativistic limit.
\end{abstract}

Available at www.worldscietific.com,:

c World Scientific Publishing Company. DOI: 10.1142/S0218301315500202 